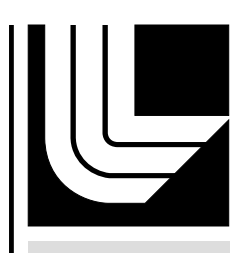

LAWRENCE LIVERM ORE N A TIO N A L LABORATORY

\title{
FREYA-a new Monte Carlo code for improved modeling of fission chains
}

C. A. Hagmann, J. Randrup, R. L. Vogt

June 15, 2012

SORMA-West 2012

Oakland, CA, United States

May 14, 2012 through May 17, 2012 
This document was prepared as an account of work sponsored by an agency of the United States government. Neither the United States government nor Lawrence Livermore National Security, LLC, nor any of their employees makes any warranty, expressed or implied, or assumes any legal liability or responsibility for the accuracy, completeness, or usefulness of any information, apparatus, product, or process disclosed, or represents that its use would not infringe privately owned rights. Reference herein to any specific commercial product, process, or service by trade name, trademark, manufacturer, or otherwise does not necessarily constitute or imply its endorsement, recommendation, or favoring by the United States government or Lawrence Livermore National Security, LLC. The views and opinions of authors expressed herein do not necessarily state or reflect those of the United States government or Lawrence Livermore National Security, LLC, and shall not be used for advertising or product endorsement purposes. 


\title{
FREYA-A new Monte Carlo code for improved modeling of fission chains
}

\author{
C. Hagmann, J. Randrup, and R. Vogt
}

\begin{abstract}
A new simulation capability for modeling of individual fission events and chains and the transport of fission products in materials is presented. FREYA ( Fission Yield Event Yield Algorithm ) is a Monte Carlo code for generating fission events providing correlated kinematic information for prompt neutrons, gammas, and fragments. As a standalone code, FREYA calculates quantities such as multiplicity-energy, angular, and gamma-neutron energy sharing correlations. To study materials with multiplication, shielding effects, and detectors, we have integrated FREYA into the general purpose Monte Carlo code MCNP. This new tool will allow more accurate modeling of detector responses including correlations and the development of SNM detectors with increased sensitivity.
\end{abstract}

Index Terms-Fission, Monte Carlo, Neutrons, Nuclear Physics

\section{INTRODUCTION}

$\mathrm{S}$ everal general purpose Monte Carlo codes (e.g. MCNP [1], TART [1], COG [1], GEANT [2]) are currently available for modeling neutron transport and fission in particular. They have in common the "average fission model" (shown in Fig. 1) meaning the characteristics of the outgoing projectiles (fission neutrons and gammas) are uncorrelated and sampled from the same probability density function (pdf). This approximation is sufficient for the calculation of average quantities such as flux, energy deposition, and multiplication. However it is unsuitable for studying correlations between neutrons and gammas from the same fission event. Experimentally, angular neutron-neutron correlations have long been established [3-7].

In recent years, various simulation treatments have made it possible to address also fluctuations of and correlations between fission observables. In particular, a Monte-Carlo approach was developed for the sequential emission of neutrons and gamma rays from an individual fission fragment

Manuscript received June 15, 2012. . This work performed under the auspices of the U.S. Department of Energy by Lawrence Livermore National Laboratory under Contract DE-AC52-07NA27344 and by Lawrence Berkeley National Laboratory under Contract DE-AC02-05CH11231.

C. Hagmann is with the Lawrence Livermore National Laboratory, Livermore, CA 94550 (phone: 925-423-6484; fax: 925-422-9560; e-mail: hagmann1@1ln1.gov).

J. Randrup is with the Lawrence Berkeley National Laboratory, Berkeley, CA 94720 (phone: 510-486-6157; fax: 510-486-4794; e-mail: randrup@ lbl.gov).

R. Vogt is with the Lawrence Livermore National Laboratory, Livermore, CA 94550 (phone: 925-423-8210; fax: 925-422-9560; e-mail: vogt2@ llnl.gov)
[8], while samples of complete fission events are provided by the code FREYA [9] used in the present work. Employing (i) nuclear data for fragment mass and kinetic energy distributions, (ii) statistical evaporation models for neutron and gamma emission, and (iii) conservation of energy and momentum, these codes are able to
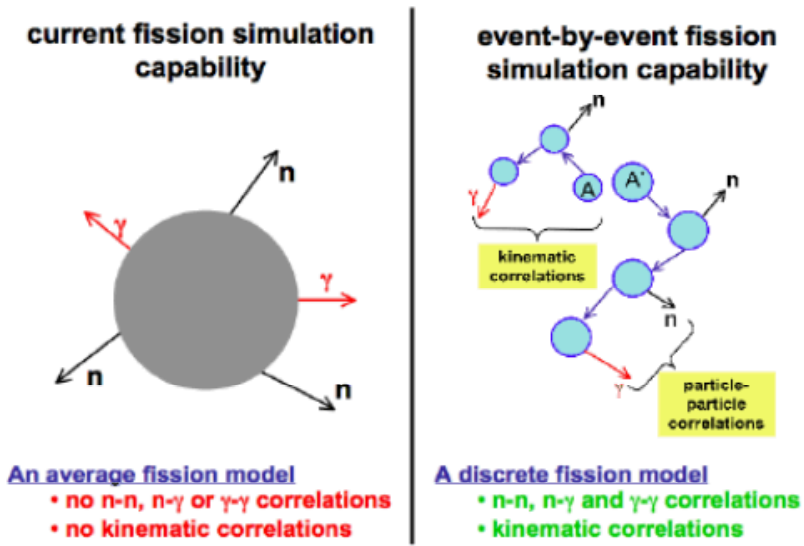

Fig. 1. The "average fission model" is used in used in conventional Monte Carlo code. FREYA treats fission in the more detailed way depicted on the right.

predict a host of correlations between outgoing fission products. These include correlations in neutron multiplicityenergy and angles, and energy sharing between neutrons and gammas. In order to model correlations in realistic detector arrays and the effects of shielding and neutron multiplication, we have integrated the standalone fission event generator FREYA into the transport code MCNP5.

\section{CODE INTEGRATION}

Upon startup, MCNP reads in the FREYA data files containing fission fragment and kinetic energy distributions of the 7 currently available fissionable isotopes: ${ }^{233} \mathrm{U}(\mathrm{n}, \mathrm{f})$, ${ }^{235} \mathrm{U}(\mathrm{n}, \mathrm{f}),{ }^{239} \mathrm{Pu}(\mathrm{n}, \mathrm{f}),{ }^{238} \mathrm{U}(\mathrm{sf}),{ }^{240} \mathrm{Pu}(\mathrm{sf}),{ }^{244} \mathrm{Cm}(\mathrm{sf}),{ }^{252} \mathrm{Cf}(\mathrm{sf})$. For each spontaneous or neutron induced fission event the code checks whether the sampled isotope is available in FREYA. If present, FREYA is called to sample multiplicity, energy, and direction of the fission neutrons, all of which are passed back to MCNP for transport. All other fission events are handled by MCNP in the usual way.

We verified that FREYA is yielding the correct average neutron induced fission spectrum within MCNP by calculating the criticality parameter $\mathrm{k}_{\mathrm{eff}}$ for the critical assemblies Godiva 
and Jezebel. Since the calculation required samples of the fission neutron energy, FREYA was called 100 times and the neutron energy was picked randomly from the samples. The fission neutron directions were sampled from an isotropic distribution. The $\mathrm{k}_{\text {eff }}$ results using FREYA were $0.9990 \pm$ 0.0006 (Jezebel) and $1.0000 \pm 0.0006$ (Godiva) and in good agreement with the default MCNP values.

\section{DETECTOR MODEL}

We adopted the MCNP detector model developed for a large array of liquid scintillators $[10,11]$ for our correlation studies. The detector consists of 64 cells of xylene $\left(\mathrm{m}_{\text {cell }}=0.5 \mathrm{~kg}\right)$, each read out by a single phototube. The cells are symmetrically arranged into octants with an array inner diameter of $\sim 60 \mathrm{~cm}$. Fig. 2 depicts the detector configuration.

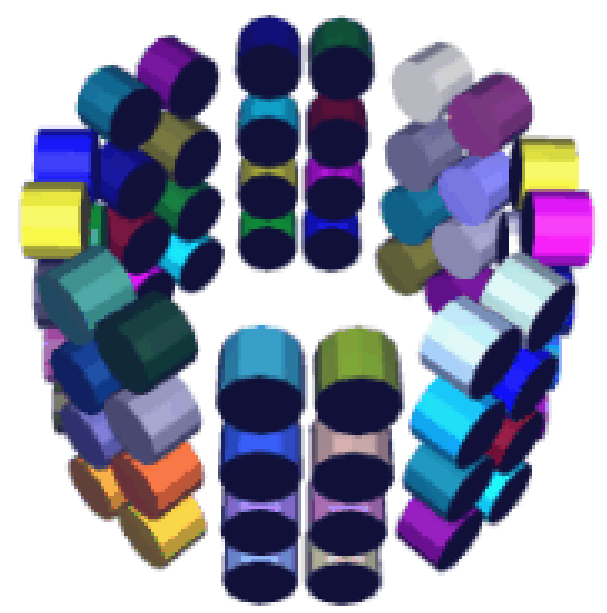

Fig. 2. Model liquid scintillator array.

The detector was designed for fast multiplicity counting and assaying of fissile material. The fast scintillator decay time (few ns) allows fast count rates compared to ${ }^{3} \mathrm{He}$ well counters. Neutron-gamma separation is accomplished by pulse shape discrimination (PSD) with a minimum proton recoil energy of $\sim 1 \mathrm{MeV}$. The detection efficiency for unmoderated fission neutrons with this threshold is $\sim 5 \%$.

\section{RESULTS}

\section{A. Multiplicity Distributions}

We studied detector responses to decays of a ${ }^{240} \mathrm{Pu}(\mathrm{sf})$ source placed at the center of the array. Cases with neutron multiplication $M>1$ were set up by placing a Pu ball $(94 \%$ $\left.{ }^{239} \mathrm{Pu}, 6 \%{ }^{240} \mathrm{Pu}\right)$ at the center, with chains initiated by ${ }^{240} \mathrm{Pu}(\mathrm{sf})$. For runs with MCNP default, we used the MCNP Watt fission spectra [12], evaluated neutron multiplicity distributions [13], and isotropic neutron emission angle. Fig. 3 shows the neutron multiplicity distribution for ${ }^{240} \mathrm{Pu}(\mathrm{sf})$ for FREYA and MCNP while Fig. 4 shows the neutron spectrum. FREYA predicts a progressively softer spectrum with increasing neutron multiplicity reflecting the decreasing fragment excitation energy as successive neutrons are evaporated. MCNP, on the other hand, only has a single "average" spectrum. These differences should result in different measured distributions due to energy dependent analysis cuts (as in fast scintillators) or cross sections (as in ${ }^{3} \mathrm{He}(\mathrm{n}, \mathrm{p})$ detectors $)$.

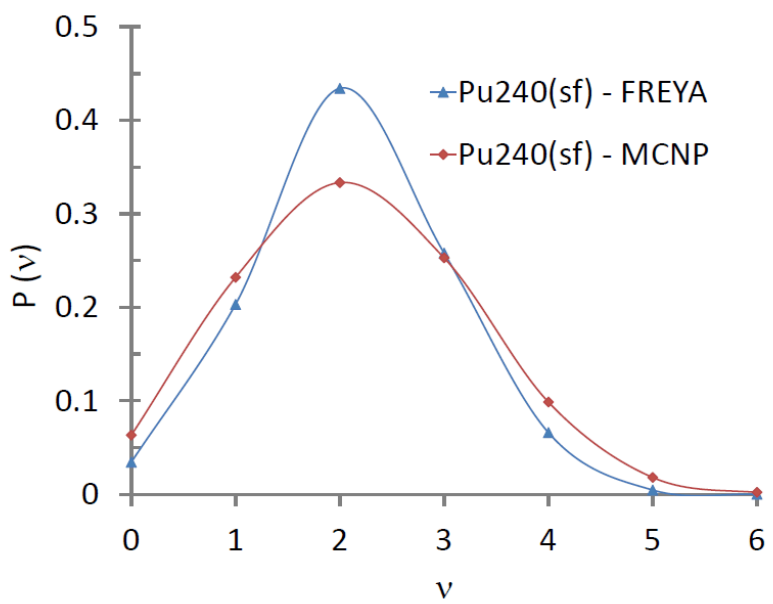

Fig. 3. The fission neutron multiplicity distribution of ${ }^{240} \mathrm{Pu}(\mathrm{sf})$ from FREYA and MCNP.

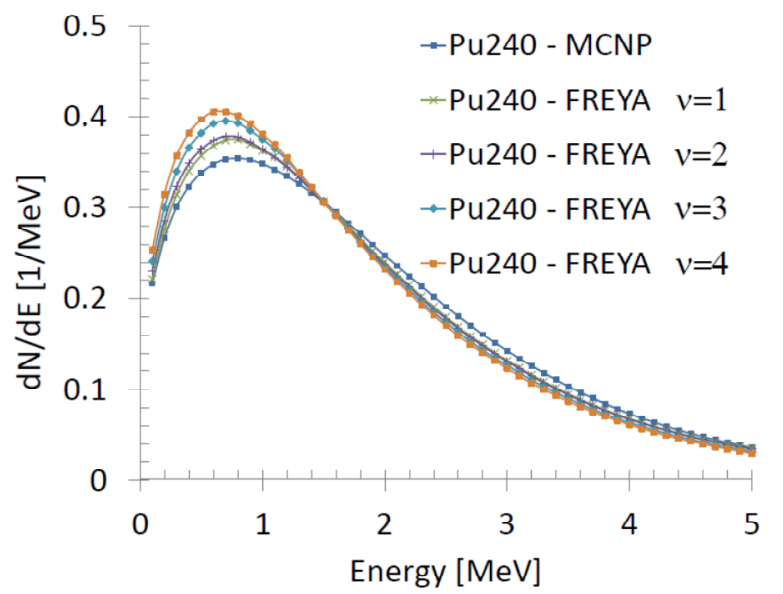

Fig. 4. The multiplicity dependent prompt fission neutron spectrum of ${ }^{240} \mathrm{Pu}$ (sf) from FREYA and the average MCNP spectrum.

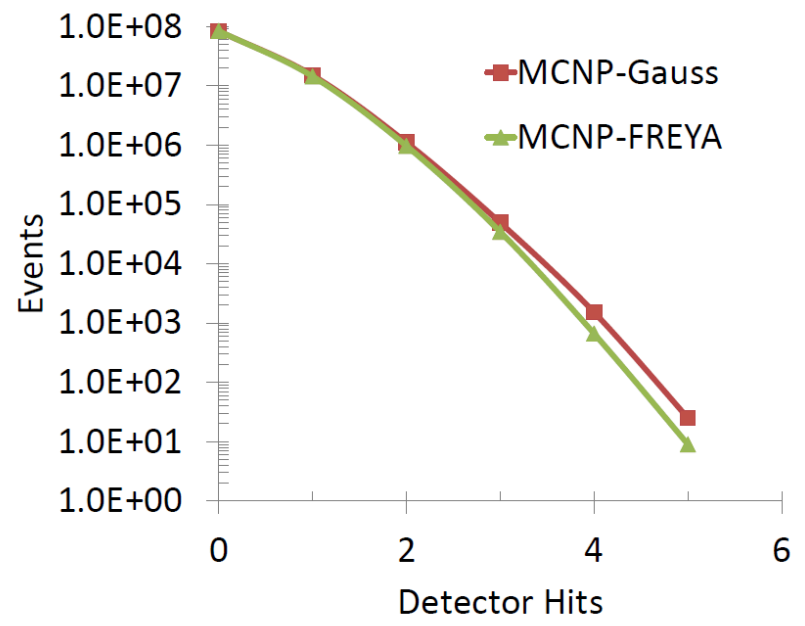

Fig. 5. Fast scintillator multiplicity distribution for a non-multiplying ${ }^{240} \mathrm{Pu}(\mathrm{sf})$ source. 


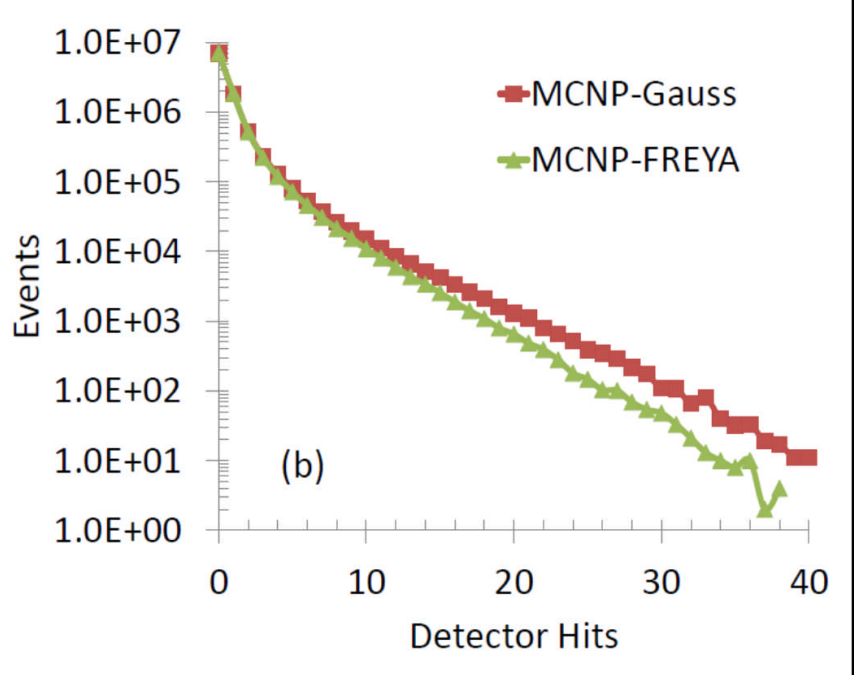

Fig. 6. Fast scintillator multiplicity distribution for a multiplying $(M=6) \mathrm{Pu}$ ball.

Fig. 5 shows the calculated detector multiplicity distributions for a pure ${ }^{240} \mathrm{Pu}(\mathrm{sf})(M=1)$, and Fig.6 the same for a multiplying $\mathrm{Pu}$ ball $(M=6)$. The number of simulated fission source events was $10^{8}$ and $10^{7}$ respectively. A detector cell was triggered if one or more proton recoils $>1 \mathrm{MeV}$ occurred in the same Monte Carlo history. In both cases FREYA predicts a smaller number of events at large multiplicities than MCNP default. An experiment to test the predictions is warranted and feasible with existing detectors and sources.

\section{B. Angular Correlations}

The second effect studied was neutron-neutron angular correlations. Almost all of the neutrons in spontaneous and low energy fission are emitted by the fully accelerated fission fragments whose back-to-back motion is imprinted on the neutron directions in the laboratory frame. Thus small angle correlations are expected from neutrons emitted from the same fragment, whereas large angle correlations arise from opposite fragments. FREYA evaporates neutrons isotropically in the center-of-mass system of the fragments and boosts them back into the laboratory frame resulting in angular correlations.

Figs. 7,8 depict the count distribution of coincidences as a function of angle for a ${ }^{240} \mathrm{Pu}(\mathrm{sf})$ source surrounded by a variable amount of iron shielding. For all $\mathrm{Fe}$ shielding thicknesses considered, FREYA shows distinct angle dependent correlations, most apparent at $90^{\circ}$ and $180^{\circ}$.

The number of events decreases with increasing shield thickness due to neutron moderation in the Fe. It is remarkable though that the ratio of events at $180^{\circ}$ to $90^{\circ}$ only decreases from $2.7(0 \mathrm{~cm} \mathrm{Fe})$ to $1.6(20 \mathrm{~cm} \mathrm{Fe})$. On the other hand, as expected, MCNP shows no correlations at large angles. The deviations from isotropy at $0^{\circ}$ stem from (i) multiple hits in a single octant by the same neutron and (ii) the requirement that each cell can be triggered only once in a fission chain.

We also investigated the effect of multiplication on the angular correlations with the source defined as in Sec. IV.A. Fig. 9 shows the expected trend of decreasing correlations with increasing $M$ due to randomization of neutron directions.

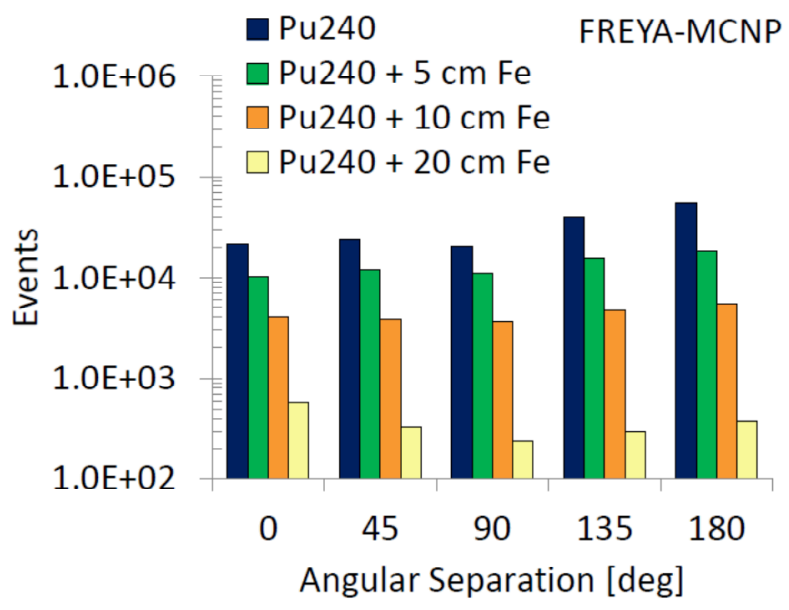

Fig. 7. Angular correlations with ${ }^{240} \mathrm{Pu}(\mathrm{sf})$ source for different thickness of iron shielding as calculated by FREYA. The number of fission events is $10^{8}$.

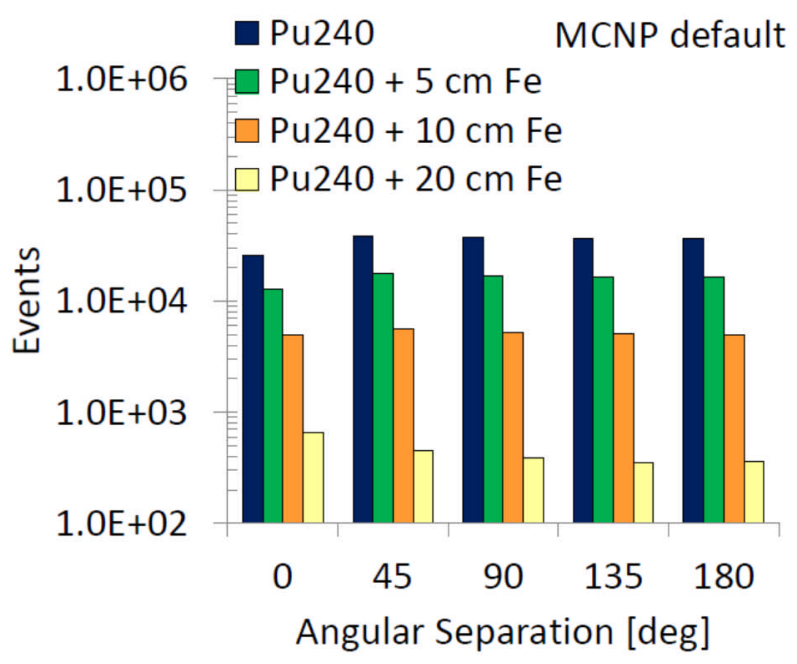

Fig. 8. As in Fig. 7 but calculated with MCNP default.

- Pu - MCNP-FREYA M=1

PU - MCNP-FREYA M=2

$\square \mathrm{PU}-\mathrm{MCNP}-F R E Y A M=6$

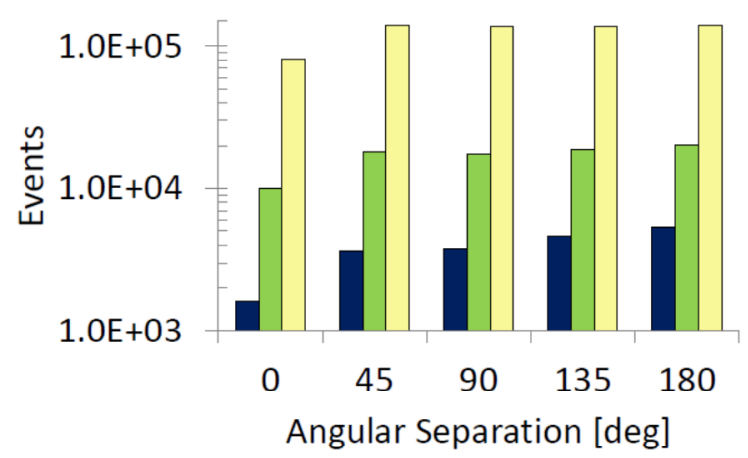

Fig. 9. Angular correlations predicted by FREYA for multiplying Pu balls. The number of fission chains for each case is $10^{7}$. 
In situations where neutron count rates are lower, as in passive HEU detection, arrays of ${ }^{3} \mathrm{He}$ counters might also be useful. We studied correlations in ${ }^{3} \mathrm{He}$ using the same array geometry but replacing the xylene material with polyethylene and a small fraction of ${ }^{3} \mathrm{He}\left(\sim 10^{-3}\right)$ and triggering on neutron capture. Figs. $10+11$ show the angular correlations for two cases of shielding

\section{Pu240 - MCNP-Gauss \\ PU240 - MCNP-FREYA}

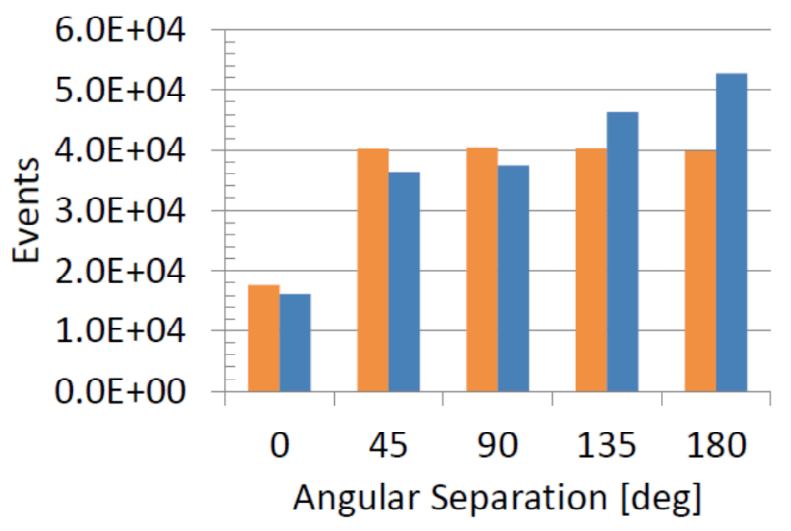

Fig.10. Angular correlations in an array of ${ }^{3} \mathrm{He}$ detectors with a ${ }^{240} \mathrm{Pu}(\mathrm{sf})$ $(M=1)$ source at the center shielding as calculated by FREYA and MCNP default. The number of fission events is $10^{8}$.

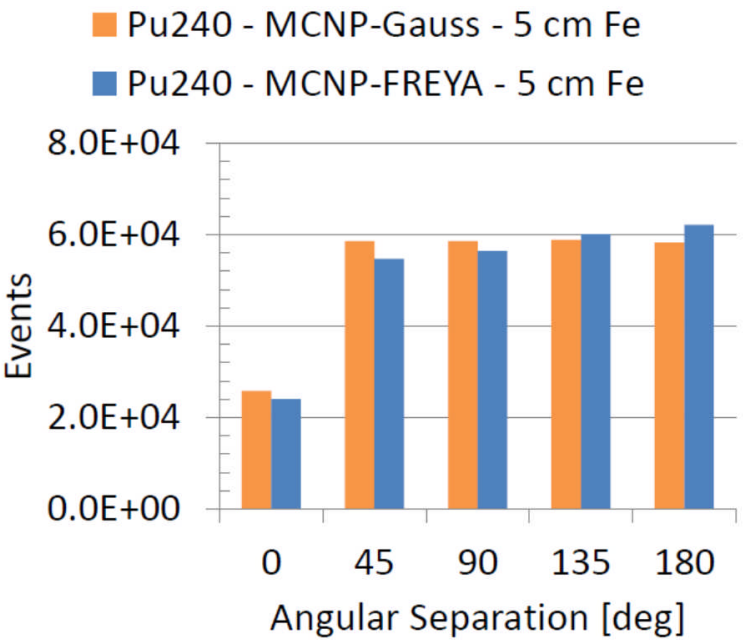

Fig. 11. As in Fig. 10 but with $5 \mathrm{~cm}$ of $\mathrm{Fe}$ shielding around the ${ }^{240} \mathrm{Pu}(\mathrm{sf})$ source.

Angular correlations are again observable at large angles for FREYA, while MCNP is angle independent. The deficit at $0^{\circ}$ is due to the requirement that each ${ }^{3} \mathrm{He}$ cell can trigger an event only once in a single fission chain.

\section{CONCLUSION}

We have integrated the event-by-event fission generator FREYA into MCNP5 for inline transport. This new capability enables the simulation of correlations that are not predicted by conventional neutron Monte Carlo codes. Neutron angular correlations are quite pronounced and should be observable in arrays of liquid scintillators or ${ }^{3} \mathrm{He}$ counters, even with substantial shielding. They provide a tell-tale signature of fission and would be useful for the rejection of cosmic neutrons.

Several improvements of FREYA are planned such as a more refined treatment of fission gammas and the addition of isotopes. We intend to make FREYA publicly available to the wider community for use in MCNP6 and GEANT4.

\section{REFERENCES}

[1] Available from the Radiation Safety Information Computational Center, http://rsicc.ornl.gov.

[2] Available from the European Organization for Nuclear Research, http://geant4.cern.ch.

[3] S. Debenedetti, J. E. Francis, Jr., W. M. Preston, and T. W. Bonner, "Angular dependence of coincidences between fission neutrons," Phys. Rev., vol. 74, pp. 1645-1650, Jun. 1948.

[4] H. R. Bowman, S. G. Thompson, J. C. Milton, and W. J. Swiatecki, "Velocity and angular distributions of prompt neutrons from spontaneous fission of ${ }^{252} \mathrm{Cf}$," Phys. Rev., vol. 126, pp. 2120-2136, Jun. 1962.

[5] J. S. Pringle and F. D. Brooks, "Angular correlation of neutrons from spontaneous fission of ${ }^{252}$ Cf," Phys. Rev. Lett., vol. 35, pp. 1563-1566, 1975.

[6] C. B. Franklyn, C. Hofmeyer, and D. W. Mingay, "Angular correlation of neutrons from thermal-neutron fission of ${ }^{235} \mathrm{U}$," Phys. Lett B., vol. 78, pp. $564-567,1978$.

[7] C. Budtz-Jørgensen and H.-H. Knitter, "Simultaneous investigation of fission fragments and neutrons in ${ }^{252} \mathrm{Cf}$ (sf)," Nucl. Phys.A, vol. 490, pp. 307-328, 1988.

[8] S. Lemaire, P. Talou, T. Kawano, M. B. Chadwick, and D. G. Madland, "Monte Carlo approach to sequential neutron emission from fission fragments," Phys. Rev. C, vol. 72, pp. 024601-1-12, 2005; "Monte Carlo approach to sequential $\gamma$-ray emission from fission fragments," ibid., vol. 73, pp. 014602-1-9, 2006.

[9] R. Vogt and J. Randrup, "Calculation of fission observables through event-by-event simulation," Phys. Rev.C, vol. 80, pp. 024601-1-11, 2009; R. Vogt, J. Randrup, J. Pruet, and W. Younes, "Event-by-event study of prompt neutrons from ${ }^{239} \mathrm{Pu}(\mathrm{n}, \mathrm{f})$," ibid., vol. 80, pp. 044611-116, 2009; R. Vogt and J. Randrup, "Event-by-event study of neutron observables in spontaneous and thermal fission," ibid., vol. 84, pp. 044612-1-14, 2011; R. Vogt, J. Randrup, D. A. Brown, M. A. Descalle, and W. E. Ormand, "Event-by-event evaluation of the prompt fission neutron spectrum from ${ }^{239} \mathrm{Pu}(\mathrm{n}, \mathrm{f})$, , ibid., vol. 85, pp. 024608-1-18, 2012.

[10] J. M. Verbeke, L. Nakae, P. Kerr, D. Dietrich, and A. Dougan, "Testing of liquid scintillator materials for gamma and neutron detection," presented at the INMN conference, Tucson, AZ, Jul. 14-15, 2009, LLNL-CONF-414602.

[11] L. F. Nakae, G. F. Chapline, A. M. Glenn, P. L. Kerr, K. S. Kim et al., "Recent developments in fast neutron detection and multiplicity counting with liquid scintillator," AIP Conf. Proc., vol. 1412, pp. 240 $248,2011$.

[12] MCNP - A general Monte Carlo N-particle transport code, version 5, Vol. I, Appendix H, LA-UR-03-1987, 2003.

[13] N.E. Holden and M.S. Zucker, "Neutron multiplicities for the transplutonium nuclides," BNL-36467, 1985; “A reevaluation of the average prompt neutron emission multiplicity (nubar) values from fission of uranium and transuranium nuclides," BNL-35513, 1985. 